\title{
RACIONALIDADE DOS AGRICULTORES: QUE DIZEM OS DADOS?
}

Eliseu Alves ${ }^{l}$

Resumo - Os dados com os quais os economistas trabalham foram gerados por agentes que tomaram decisões baseadas no conhecimento que tiveram dos mercados, de tecnologias e de como se comportam perante o risco. Numa economia competitiva, o mercado induz à busca da máxima eficiência, que se traduz na minimização de custo, quando o nível de produção é fixado, ou na maximização da renda líquida, quando se deixa aquela restrição de lado. É claro que, no nível de produção correspondente à renda líquida máxima, o custo é também mínimo. Assim, é pertinente perguntar se os dados revelam que os produtores minimizam custo ou maximizam a renda líquida, e que implicações isto tem na existência da função custo e de produção. Este trabalho procura responder a estas questões, numa exposição em que se realçam conceitos, deixando de lado a demonstração de teoremas.

Palavras-chave: Custo, renda líquida, agricultura.

\section{Introdução}

O caminho comum de quem quer conhecer a função de produção ou de custo é estimar, diretamente, uma fórmula previamente escolhida e verificar o comportamento estatístico desta.

Os dados foram gerados pelos produtores, de acordo com as regras de comportamento que seguem, com o conhecimento que têm dos métodos de produção, dos preços de produtos e insumos, e, ainda, obedecendo-se às restrições que não podem remover.

É possível que as observações sejam incompatíveis com a função custo ou de produção; assim, os resultados estatísticos do modelo padecem de um vício de origem, que será discutido neste trabalho.

${ }^{1}$ Eliseu Alves é pesquisador da Embrapa.

Recebido em 05/11/2003 Aceito em 01/12/2003 
Na prática, os dados não respeitam totalmente as condições teóricas, embora seja possível perturbá-los, de modo que se conformem com elas. A questão é saber qual é a magnitude aceitável para a perturbação e que critérios devem ser obedecidos. Esta é outra questão que se aborda.

De que racionalidade se fala? Minimizar custo é um critério, e outro é maximizar a renda líquida, mas procura-se alguma condição que permita restringir-se a um conjunto finito de observações, ou seja, embora possa ser trabalhoso, por inspeção pode-se descobrir o que ocorreu.

Esta linha de pesquisa começou com Afriat $(1967,1972,1976)$ e foi aperfeiçoada por Varian $(1982,1983,1984,1985)$. Houve extensão para insumos fixos, por Ray eBhadra (1993), e para situações que envolviam risco, por Chavas e Cox (1993). Em Alves (2000), os trabalhos de Varian, Ray e Bhadra, e de Chavas e Cox foram revistos, e todos os teoremas foram demonstrados.

\section{Pressuposições}

Admite-se a existência de um conjunto de produção que descreve a tecnologia que é do conhecimento de todos os produtores que geraram as observações. Se cada produtor tivesse um conjunto de produção que lhe é específico, as comparações entre produtores perderiam a razão de ser. A essência do procedimento é a comparação entre agricultores.

Representa-se por x uma cesta de insumos, e por y, a produção, que pode ser múltipla. No caso, $y \geq z$ significa que cada componente de y é maior ou igual a cada componente de z. Em muitos casos, restringir-seá a produção de um único produto; $x$ é um vetor de n componentes e y, de m componentes. As componentes de x e y são não-negativas. Na maioria dos casos estudados, $m=1$, ou seja, produz-se um único produto. Reserva-se o símbolo * para indicar a multiplicação escalar de dois vetores, como em $w^{*} x$. 
Simbolicamente, representa-se o conjunto de produção, da seguinte forma: $V(y)=\{x: x$ produz $y\}$.

Um exemplo muito restrito de $\mathrm{V}(\mathrm{y})$ é uma isoquanta. $\mathrm{V}(\mathrm{y})$ satisfaz a duas pressuposições:

1. Uma família de conjunto de produção é encadeada se:

$V\left(y^{i}\right) \geq V\left(y^{i}\right)$, então, $V\left(y^{i}\right) \leq V\left(y^{i}\right)$,

ou seja, se uma combinação de insumos produz determinado produto, ela produzirá qualquer quantidade menor dele. Os conjuntos de produção que produzem maior quantidade de produto ficam, portanto, dentro dos conjuntos de produção que produzem menos. Para produzir a quantidade y, o agricultor tem muitas opções de conjuntos de produção, e não apenas a daquele que lhe é específico, como, por exemplo, todos os conjuntos que produzem mais que $y$.

Por que os conjuntos são encadeados?

Se $y^{1} \leq y^{2} \leq \ldots \leq y^{n}$, segue-se que $V\left(y^{n}\right) \leq V\left(y^{n-1}\right) \leq \ldots \leq V\left(y^{2}\right) \leq V\left(y^{1}\right)$. Desta forma, a cadeia de conjuntos dá aos produtores várias opções de escolha, quanto aos conjuntos, e quebra-se, assim, a especificidade.

2. Não se pode coibir o desperdício: os agricultores podem desperdiçar insumos, sem incorrer em nenhum custo. Quem o evitará é o mercado, ao eliminar os agricultores ineficientes. Simbolicamente, se $x \in V(y)$, e $u \geq x$, então, $u \in V(y)$. Assim, se uma combinação de insumos produz $\mathrm{y}$, então, toda combinação que tiver todas as componentes maiores ou iguais à inicial também produzirá y. Sem esta pressuposição, o papel do mercado para gerar eficiência técnica desaparecerá ${ }^{2}$.

${ }^{2} \mathrm{~A}$ combinação de insumos que produz y pertence à respectiva isoquanta. 


\section{Racionalização de custo}

No caso, produz-se um único produto. Quando é que o agricultor A é racional no que diz respeito a custo? Quando a combinação de insumos que ele escolheu para produzir y não custar mais que qualquer outra combinação que produza y, avaliada pelos preços pagos pelo agricultor A, ou seja, pelos preços que A pagou pelos insumos, nenhuma combinação dos demais agricultores, ou qualquer outra que produza a mesma quantidade, pode custar menos. Em símbolos, c-racionalização, e c representa custo,

\section{Definição 1 (c-racionalização)}

Se $x^{i}$ é a combinação de insumos que o agricultor escolheu para produzir $y^{i}$, e se x é outra combinação de insumos que produza a mesma quantidade, também de seu conhecimento, ou seja, $x \in V\left(y^{i}\right), w^{i}$ o vetor de preços pagos pelos insumos pelo agricultor $\mathbf{i}$, então,

$$
w^{i} * x^{i} \leq w^{i} * x, x \in V\left(y^{i}\right) .
$$

Nota-se que a definição é típica de minimização de custo. No entanto, não se realiza nenhum exercício de minimização para se encontrar $x^{i}$, pois se admite que o agricultor tenha feito a escolha correta. Por isto, não se importuna com os teoremas de existência. $\mathrm{O}$ que o teorema seguinte fará, a fim de saber se o produtor é racional, é reduzir a procura aos pontos observados, sem ter que verificar cada ponto de $V\left(y^{i}\right)$. Aí está o seu poder. 


\section{Teorema 1}

As seguintes condições são equivalentes:

(i) Existe uma família encadeada de conjuntos de produção, $V(y)$, em que c-racionaliza as observações;

(ii) Se $y^{j} \geq y^{i}$,então, $w^{i} * x^{j} \geq w^{i} * x^{i}$;

(iii) Existe uma família não-trivial de conjuntos de produção, que são convexos, fechados, encadeados e permitem o desperdício.

O item (ii) diz que quem produz mais não pode gastar menos preços dos insumos de quem produz menos. Este item é verificável empiricamente; assim, é possível saber se os produtores da amostra são minimizadores de custo e se a família de conjuntos nos quais se busca o mínimo também existe. Varian (1985), ao sugerir um teste para saber se as discrepâncias observadas levam à rejeição da hipótese de c-racionalização, aplicou-o a dois conjuntos de dados. No Brasil, Souza e Alves (2003) aplicaram o teste a uma amostra de produtores de leite.

Se o item (ii) passar no teste, o item (iii) segue-se. Por ele, o conjunto $\mathrm{V}(\mathrm{y})$ garante a existência da função custo, que, então, poderá ser estimada, ou seja, os dados da amostra não são incompatíveis com a existência de uma função custo. Então, os dados indicam que é legítimo estimar a função custo.

Se o item (ii), do teorema, passar pelo teste, pelo item (i), os produtores são minimizadores de custo.

\section{C-racionalização e existência de função de produção}

O teorema da dualidade ensina como se obter a função de produção da função custo e vice-versa. É, assim, natural indagar a quais condições os dados devem satisfazer, de modo que impliquem a existência de uma função de produção. 
Definição 2 (cf-racionalização)

A função de produção, definida em $R_{+}{ }^{n}$, cf-racionalizará os dados $\left(w^{i}, x^{i}, y^{i}\right)$, conforme o critério de minimização de custo, se:

(i) $f\left(x^{i}\right)=y^{i}$;

(ii) $f(x) \geq f\left(x^{i}\right) \rightarrow w^{i} * x \geq w^{i} * x^{i}$.

Requer-se que o gráfico da função passe por todas as observações e que quem produza mais não possa gastar menos. Essa exigência é compreensível por causa de (ii), mas cria problemas estatísticos para quem quiser estimar $\mathrm{f}(\mathrm{x})$, pois o ajustamento tem que ser perfeito.

\section{Teorema 2}

As seguintes condições são equivalentes:

1. Existe uma função contínua que cf-racionaliza as observações;

2. $y^{j} \leq y^{i} \rightarrow w^{j} * x^{j} \leq w^{j} * x^{i}$;

3. $y^{j}<y^{i} \rightarrow w^{j} * x^{j}<w^{j} * x^{i}$;

4. Existe uma função contínua semicôncava e monótona crescente, a qual cf-racionaliza as observações ${ }^{3}$.

Em relação ao teorema 1, tem-se que checar, além de 2, a condição 3: quem produz menos tem que gastar menos do que quem produz mais. $\mathrm{O}$ teste de Varian (1985) aplica-se sem maiores dificuldades, e, por 4, a função de produção é semicôncava contínua. A função $\mathrm{f}(\mathrm{x})$ será semicôncava, se $f(x) \geq a$ e $f(z) \geq a \rightarrow f(t x+(1-t) z) \geq a, 0 \leq t \leq 1$.

\footnotetext{
${ }^{3} x \geq z \rightarrow f(x) \geq f(z)$, implica que a função é monótona crescente.
} 


\section{Retorno constante: cf-racionalização}

Pelo critério da cf-racionalização é possível testar se a função de produção admite retornos constantes à escala, e se a condição obtida não é complicada de se verificar empiricamente. Por definição, a função de produção admite retornos constantes à escala, linear homogênea; quando se duplicarem os insumos, a produção se duplicará também. Tecnicamente, $f(t x)=t f(x) t \geq 0$. Define-se $v_{i}=\frac{w_{i}}{w^{*} x}$. Assim, a componente de preço normalizada $v_{i}$ é preço do insumo $\mathbf{i}$, dividido pelo custo de produção, $w^{*} x$; e $v$ é o vetor normalizado de preços.

\section{Teorema 3}

1. Existe uma função de produção linear homogênea que cfracionaliza os dados;

2. $v^{j} * x^{i} \geq \frac{y^{i}}{y^{j}}$ para todo $i j$;

3. Existe uma função de produção contínua, monótona crescente e linear homogênea que cf-racionaliza os dados.

A condição 2 é, empiricamente, verificável. Fixa-se o agricultor i, então, haverá $\mathrm{n}$ testes, quando $\mathrm{n}$ for o tamanho da amostra, porque variarão os v's e os y's dos outros agricultores e o próprio $v^{i} * x^{i}=1$.

É possível estimar uma função de produção, que, pelos resultados estatísticos, deve ser linear homogênea. Mas, se a condição 2 for violada, estar-se-á diante de resultado enganoso. Mas, e se as violações forem pouco freqüentes? Aí o pesquisador terá de usar seu julgamento a respeito do que é pouco e confrontá-lo com o ajustamento da função de produção. 
Há uma condição para verificar se a função de produção é homotética, mas ela é complicada de se aplicar aos dados, por isto, é omitida.

\section{Existência da função de produção: o critério da renda líquida}

É interessante usar a renda líquida em conjugação com a função de produção, como critério de racionalização, no caso rf-racionalização.

Definição 3 (rf-racionalização)

Os dados serão rf-racionalizáveis, se:

1. $f\left(x^{i}\right)=y^{i}$,

2. $p^{i} * y-w^{i} * x \leq p^{i} * y^{i}-w^{i} * x^{i}$.

A condição 2 diz que o agricultor escolheu a combinação que maximiza a renda líquida. Note-se que a renda líquida será limitada por aquela do agricultor i, quando forem usados os seus preços para produto e insumos na avaliação.

\section{Teorema 4}

1. Existe uma função de produção que rf-racionaliza os dados, 2.

3. $p^{i} * y^{i}-w^{i} * x^{i} \geq p^{i} * y^{j}-w^{i} * x^{j}$, Existe uma função de produção, definida em contínua, côncava e monótona crescente que rf-racionaliza os dados.

A condição 2 diz que, considerando-se o preço do produto e o vetor preço dos insumos, a combinação produto-insumo do agricultor $i$ é melhor que qualquer outra combinação escolhida pelos outros produtores, quanto à renda líquida. A condição 2 pode ser verificada empiricamente e assegura, em 3, a existência de uma função da função de produção, com as propriedades mencionadas. O problema estatístico é que o gráfi- 
co da função de produção, no caso côncava e monótona crescente, tem que passar por todas as observações. Assim, o ajustamento tem que ter $\mathrm{R}^{2}=1$, ou, estatisticamente, não pode divergir disto. Em 1, a condição 2 assegura que os agricultores maximizem a renda líquida e sejam racionais nesse ponto de vista.

Pelo critério da rf-racionalização, é possível testar se a função de produção é separável, mas as condições são muito complicadas de serem aplicadas aos dados. Por isto, omite-se o enunciado do teorema pertinente, que está em Alves (2000).

\section{Produção múltipla e r-racionalização}

Exige-se uma mudança de símbolos. O conjunto Y contém dois conjuntos de vetores; os de sinal não-negativo representam a produção e os de sinais não-positivos, os insumos. Há $n$ insumos e m produtos, portanto, $Y$ tem $n+m$ componentes. $O$ vetor $p$ tem $n+m$ componentes, para representar os preços de $\mathrm{n}$ insumos e m produtos. Os preços são nãonegativos. Portanto, se $x \in Y, p * x$ será a renda líquida.

As observações serão r-racionalizadas, se $p^{i} * x^{i} \geq p^{i} * x, x \in Y$, ou seja, a combinação escolhida pelo agricultor i, quando ela for avaliada pelo seu vetor preço, é a que maximiza a sua renda líquida.

\section{Teorema 5}

1. Existe um conjunto $Y$ que r-racionaliza as observações;

2. $0=p^{i} * y^{i} \geq p^{i} * y^{j} \quad j=1,2 . . T$ e $T$ é o tamanho da amostra;

3. Existe um cone convexo e fechado, $Y$, e $z$ pertence a $Y$ e $y \leq z$, então, y pertence a $Y$ e $Y$ r-racionaliza as observações. 
O significado de $y \leq z$ merece explicação. As componentes de y, que dizem respeito à produção, são menores ou iguais às respectivas componentes de z. As componentes de y, que se referem a insumos, são maiores ou iguais, em valores absolutos, às respectivas componentes de z, ou seja, y produz menos ou a mesma coisa e gasta mais ou igual a z. Assim, permite-se o desperdício.

Por 3, Y é um cone, ou seja, se z pertence a $Y$, segue-se que $t z$, t é um número real não-negativo, pertence a Y. Por ser Y um cone, a renda líquida máxima tem que ser zero.

A condição 2 é empiricamente verificável. Se passar no teste, segue-se que os agricultores maximizam a renda líquida e são r-racionais. Como 3 segue-se de 2, pode-se, legitimamente, estimar uma função custo para produtos múltiplos.

\section{Teste de hipótese}

As observações em número de $\mathrm{T}$, admitindo-se um único produto, correspondem a $\left(w^{i}, x^{i}, y^{i}\right) i=1,2, \ldots, n ;$ i particulariza o agricultor $\mathbf{i}$; w é vetor de preços; x é o vetor de insumos; e y é a produção.

Pelo teorema 1, condição (ii), $y^{i} \leq y^{j} \rightarrow w^{i} * x^{i} \leq w^{i} * x^{j}$, e o teste estatístico se faz por meio desta.

Em primeiro lugar, ordenam-se os y's do menor para o maior. Não havendo empates, o número de comparações é dado por $\mathrm{T}^{*}(\mathrm{~T}-1) / 2$. Se houver empate, é preciso comparar $w^{i} * x^{i} \operatorname{com} w^{i} * x^{j}$, e $w^{j} * x^{i}$ com $w^{j} * x^{j}$. O sinal da desigualdade tem que ser o mesmo. A cada acerto atribui-se 1 e, a cada erro, 0 . Se o número de acertos for grande, acima de $80 \%$, em relação ao total de comparações, será um primeiro sinal que não desfavorece a hipótese. Varian (1985) afirmou 
que o número de comparações é $T^{2}$, porque ele olha a desigualdade $y^{i} \leq y^{j}$ nas duas direções. Mas, a condição dois não implica bem isto. Se os insumos forem medidos em unidades monetárias, o problema desaparecerá, como ocorreu em Souza e Alves (2003).

A base do teste formal é admitir que existe quantidade correta dos insumos e que o agricultor não a usou por causa fatores aleatórios, ou seja, $x_{i k}=z_{i k}+\varepsilon_{i k}$ e k refere-se a insumo e i, ao agricultor; k varia de 1 a $\mathrm{M}$; e $\mathrm{i}$, de 1 a T. $\mathrm{O}$ vetor $\mathrm{x}$ tem $\mathrm{n}$ componentes e $\mathrm{z}$ representa a demanda verdadeira, que é desconhecida. Admite-se que $\varepsilon_{i k}$ seja normal e independentemente distribuído, com média zero e mesma variância, $\sigma^{2}$.

Caso fosse possível observar a verdadeira demanda z, poder-se-ia computar a estatística $V=\sum_{i=1}^{T} \sum_{k=1}^{M}\left(z_{i k}-x_{i k}\right)^{2} / \sigma^{2}$, que, pelas hipóteses feitas, tem distribuição qui-quadrado com TM graus de liberdade.

A estatística V não é observável, mas pode-se calcular o seu limite inferior pela minimização da soma de quadrado do numerador, respeitandose as restrições, $w^{i} * x^{i} \leq w^{i} * x^{j} i, j=1,2, \ldots n$. Seja R o valor obtido pela solução da programação quadrática, o que isto significa? Ora, se as restrições fossem satisfeitas, o mínimo buscado seria zero. O que se procura fazer, quando algumas restrições não são satisfeitas, é modificar, o mínimo possível, as observações sobre os insumos, para que as restrições sejam atendidas. É fácil compreender que o problema de programação quadrática tem restrições lineares e corresponde a mínimos quadrados com restrições.

Assim, $R / \sigma^{2} \leq V$. Usando-se $\mathrm{R}$, obtém-se um $\chi^{2}$ nunca maior que o dado por $\mathrm{V}$, e aumenta-se a chance de rejeitar a hipótese de que os produtores minimizam custo. 
Mas a variância $\sigma^{2}$ é desconhecida, e não se pode calcular o qui-quadrado. A solução proposta por Varian (1985) é obter o qui-quadrado, que corresponde a TM graus de liberdade, e nível de significância de rejeição de hipótese acordado. Seja este valor igual $K_{\alpha}$; assim, $\sigma^{2}=R / K_{\alpha}$. Que se faz com este valor? Aí o pesquisador tem que exercer o seu julgamento para saber se ele é suficientemente pequeno, como erro de medição. A estatística $\bar{\sigma} / T M$ é utilizada por Varian para saber se o desvio-padrão médio, em relação a cada insumo e produtor, é pequeno.

Em virtude de as unidades de medidas dos insumos serem diferentes, na aplicação que fez, Varian (1985) especificou o termo do erro, como segue: $z_{i k}=x_{i k}\left(1+\varepsilon_{i k}\right)$.

Varian (1985) respondeu a uma série de objeções ao método e recomendou que o leitor as lesse.

\section{Perturbação dos dados e eficiência econômica}

A programação quadrática fornece a cesta de insumos de cada produtor, de modo que ela obedeça às restrições expressas em (ii), do teorema 1. Considerando-se os novos valores dos insumos, pode-se calcular o custo a que eles correspondem e dividir o valor obtido pelo custo observado. Tem-se, assim, medida de eficiência custo, subordinada ao fato de que a combinação de insumos da programação quadrática é compatível com a idéia de que os produtores sejam minimizadores de custo. A orientação estudada é insumos, mas é fácil adaptá-la para produto (Souza e Alves, 2003).

Há outro método para calcular a eficiência custo, qual seja, o encapsulamento de dados (DEA), com orientação produto ou insumo. Com testes de hipóteses, uma exposição rigorosa do método pode ser encontrada em Souza, Geraldo S. (2003). A nova combinação do DEA, 
necessariamente, não necessita satisfazer à condição (ii), do teorema 1, e a modificação dos dados tende a ser maior que a do método Varian.

Um dos usos das medidas de eficiência econômica é ajudar os agricultores a administrar bem seu negócio. Mas, quanto maior for a perturbação dos dados pela técnica de programação, mais difícil se tornará a aceitação da nova combinação de insumos. A esse respeito, o método Varian se destaca, porque visa à menor perturbação de cada observação, respeitando-se a condição (ii), do teorema 1. Assim, um critério de seleção de métodos de análise da eficiência econômica é o grau de perturbação das observações, medido pela soma dos quadrados das diferenças entre o valor observado e o valor proposto.

A boa administração objetiva aumentar a renda líquida do estabelecimento. Assim, o grau de aumento da renda líquida precisa ser levado em conta. Como o DEA procura reduzir, o máximo possível, a quantidade de cada insumo, ele tende a levar vantagem sobre o método de Varian, mas é preciso checar se sua recomendação de consumo de insumos não escapa das possibilidades dos produtores.

Em Souza e Alves (2003), o método de Varian comportou-se muito bem, quanto aos graus de perturbação da combinação de insumos. Mas, quanto à renda líquida, perdeu para o DEA, mas não deixou de trazer aumento substancial.

\section{Conclusões}

O trabalho mostrou que é importante verificar se os dados são compatíveis com a existência da função custo, ou de produção, e ainda indicou como testar a hipótese de que os agricultores sejam minimizadores de custo.

Discutiram-se dois critérios para comparar métodos que medem a eficiência custo: perturbação dos dados e aumento da renda líquida. O método Varian comportou-se bem em relação aos dois critérios e teve a van- 
tagem de propor uma combinação de insumos que é compatível com a minimização de custos e, ao mesmo tempo, não diverge tanto daquela correspondente às observações. Por isto, é mais facilmente aceitável pelos produtores.

É preciso aperfeiçoar a parte estatística do método de Varian e estudar melhor os critérios que permitem julgar os diferentes métodos que medem a eficiência custo.

\section{Referências bibliográficas}

AFRIAT, S. N. The construction of a utility function from expenditure data. International Economic Review, 8, 67-77, 1967.

AFRIAT, S. N. Efficiency estimation of production functions. International Economic Review, 13, 568-598, 1972.

AFRIAT, S. N. Combinatorial Theory of demand. London, InputOutput Publishing Company, 1976.

ALVES, Eliseu Teoria da produção: métodos não paramétricos (mimeo.), Brasília, Embrapa, 2000.

CHAVAS, Jean-Paul e COX, Thomas L. On genarilized revealed preference analysis. The Quarterly Journal of Economics, 108, 493506, 1993.

RAY, S. C. e BHADRA, D. Nonparametric test of cost minimizing behavior: a study of Indian farmers. American Journal of Agricultural Economics, 75, 4, 990-999, 1993.

SOUZA, Geraldo da Silva. Funções de produção: uma abordagem estatística com o uso de modelos de encapsulamento de dados, Brasília, Embrapa, Texto para Discussão 17, 2003. 
SOUZA, Daniel, P. H. \& ALVES, Eliseu. Dois critérios para avaliação da eficiência técnica, Revista de Economia e Agronegócio, Vol.1, No. 3, 2003.

VARIAN, H. The nonparametric approach to demand analysis, Econometrica, 50, 945-974, 1982.

VARIAN, H. The nonparametric test of consumer behavior, Reviw of Economic Studies, 50, 99-110, 1983.

VARIAN, H. The nonparametric approach to production analysis, Econometrica, 52, 579-597, 1984.

VARIAN, H. Nonparametric analysis of optimizing behavior with measurement error. Journal of Econometrics, 30, 445-458, 1985. 
REVISTA DE ECONOMIA E AGRONEGÓCIO, VOL.1, No 4 